Proceedings

\title{
Conceptual Design of a New Multi-Component Test Bench for the Dynamic Characterization of Running Specific Prostheses ${ }^{+}$
}

\author{
Nicola Petrone 1,*, Gianfabio Costa 1, Gianmario Foscan 1, Francesco Bettella 1, \\ Gianluca Migliore ${ }^{2}$ and Andrea Giovanni Cutti ${ }^{2}$ \\ 1 Department of Industrial Engineering, University of Padova, Via Venezia 1, 35131 Padova, Italy; \\ gianfabio.costa@gmail.com (G.C.); gianmario.foscan@gmail.com (G.F.); fra.bettella@gmail.com (F.B.) \\ 2 INAIL, Budrio, Via Rabuina, 14, 40054 Vigorso BO, Italy; gi.migliore@inail.it (G.M.); \\ ag.cutti@inail.it (A.G.C.) \\ * Correspondence: nicola.petrone@unipd.it; Tel.: +39-049-827-6761 \\ + Presented at the 13th Conference of the International Sports Engineering Association, Online, \\ 22-26 June 2020.
}

Published: 15 June 2020

\begin{abstract}
Stiffness properties of running specific prostheses (RSP) for Paralympic runners are fundamental in the selection of the optimal running prosthetic foot (RPF) for sprint and jump events, depending on the athlete's anthropometry and characteristics. RPFs are J-shaped or C-shaped, clamped to the socket or the pylon of the prosthetic leg. The aim of this work was to develop a test bench suitable for the static and dynamic characterization of a running prosthetic feet (RPF). Based on the evidence that the ground reaction force components change their relative orientation to the pylon or socket during the stance, loads were resolved in the socket reference frame and a multicomponent test bench was designed and constructed. Two perpendicular actuators can apply static and dynamic loads to the foot while contacting a surrogate ground inclined at different angles. The preliminary tests show how the alignment, load combination, and ground angle can affect RPF stiffness curves.
\end{abstract}

Keywords: running specific prostheses; test bench; static and dynamic stiffness

\section{Introduction}

Running specific prostheses (RSP) are a fundamental aid for Paralympic sprint runners and long jumpers. As their introduction, researchers have recognized that properties of a running prosthetic foot (RPF) are influencing the behavior of athletes due to their interaction with the ground and the effects on the athlete's feeling, comfort, and overall performance [1-5]. Together with the alignment between socket, knee (in trans-femoral), and foot, the intrinsic stiffness of RPFs is a key factor for the fitting of the prosthesis in sprint and jump, depending on the athlete's anthropometry and running characteristics [6]. Several studies have been focusing on the static stiffness of RPFs after equipping typically universal test machines with upper clamps for the support of the RPF and introducing fixed or sliding devices for the simulation of the ground floor at the RPF tip, at different ground angles and with different restraint conditions [7-9].

RPFs are typically J-shaped or C-shaped, clamped to the socket or the pylon of the prosthetic leg. Loads acting on the RSP come from ground reaction forces (GRF) and reach the leg through a complex combination of loads parallel and normal to the prosthetic pylon/socket assembly. Most literature focused on the collection of GRFs has been conducted indoors with elite athletes performing their sprint on a set of force platforms embedded in the floor of a Motion Analysis Laboratory [10]. 
The aim of this work was to develop a test bench suitable for static and dynamic characterization of RSPs, with special attention on the RPF: In particular, the bench allows the simultaneous application of loads parallel and perpendicular to the foot clamp axis at different angles to the ground.

\section{Materials and Methods}

The bench was conceived and designed in such a way that the combination of forces parallel and normal to the pylon or socket can be applied independently to the largest variety of RSPs and RPFs while changing the relative angle between ground and RPF. Steps followed in the process were (i) reanalysis of literature data to estimate loads on the foot, (ii) concept, (iii) design, (iv) construction, and (v) testing of the bench.

The GRF components change their relative orientation to the pylon or socket during the stance as the athlete's leg completes the step, as shown in Figures 1 and 2, where literature data collected in the global reference frame [10] were resolved in the socket reference frame.

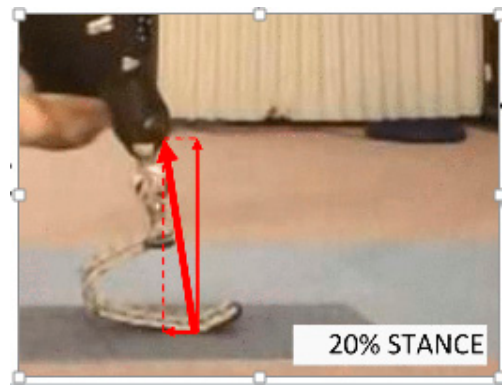

(a)

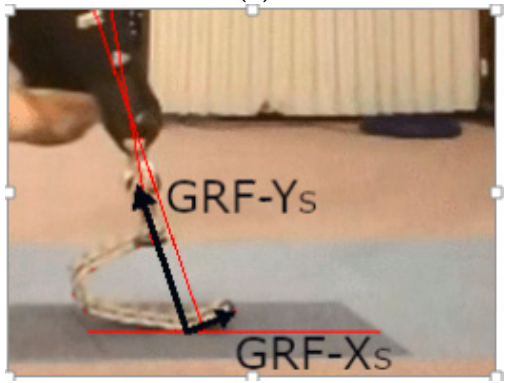

(c)

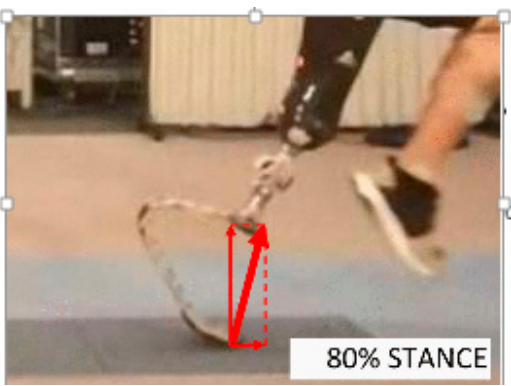

(b)

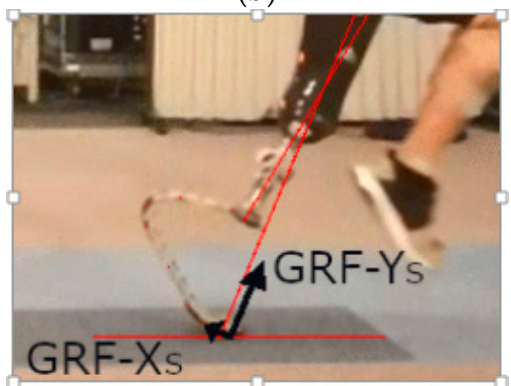

(d)

Figure 1. Frames showing ground reaction force (GRF) components in global reference frame for an elite sprinter, from [10]: (a,b) Global components at 20\% and $80 \%$ stance; (c,d) GRF resolved in the socket reference frame for the same instants of $20 \%$ and $80 \%$ stance.

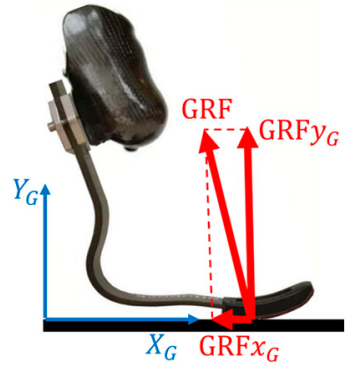

(a)

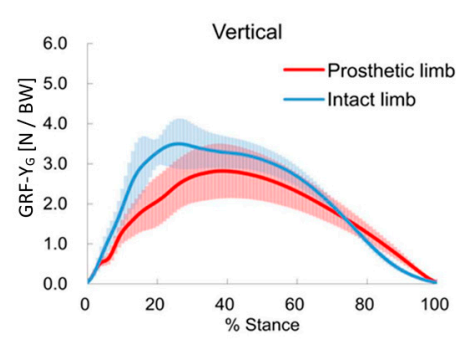

(b)

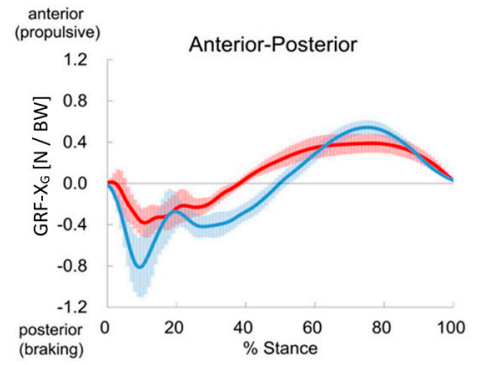

(c) 


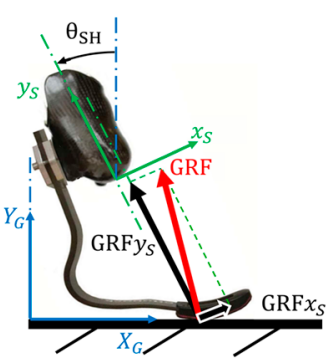

(d)

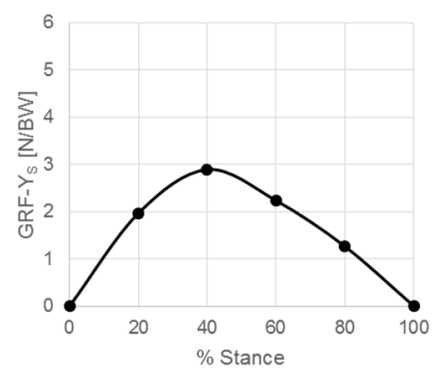

(e)

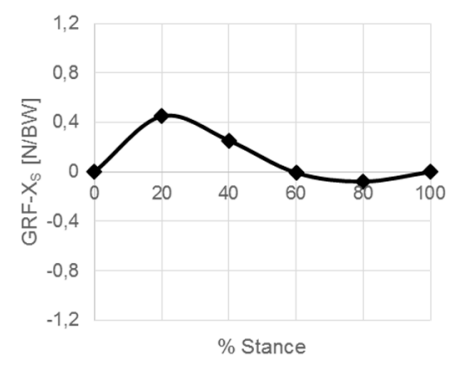

(f)

Figure 2. (a-c) GRF components in global reference frame for an elite sprinter [10]: (d-f) GRF resolved in the socket reference frame for all stance phases.

A vertical double portal frame was fixed to a solid cast iron basement: Two servo-hydraulic cylinders (MTS 244, $15 \mathrm{kN}, 200 \mathrm{~mm}$ ) were applied to the frame (Figure 3). The vertical actuator can move a slide to which the RSP is rigidly fixed by means of a 6-axis load cell (ME, K6D80/2 kN/100 $\mathrm{Nm})$. The tip of the RSP contacts a triaxial load cell (ME, K3D120 $\pm 5 \mathrm{kN} / \mathrm{VA}$ ) covered with tartan, mounted on a second slide that a second actuator can move horizontally. The orientation of the plane can vary between $+/-45^{\circ}$, simulating mutual orientations of the RSP and ground. The vertical slide is equipped with four linear bearings designed to sustain the high dynamic and impact reaction loads generated at the surrogate ground by the RPF tip and transmitted to the slide, while ensuring low friction and high dynamic stiffness. The horizontal slide is again equipped with four linear bearings designed to sustain the high loads generated at the surrogate ground by the RPF tip. In addition, due to the connection with the hydraulic actuator, the horizontal slide can be fixed or can be free of sliding in the horizontal direction as demanded by the RPF stiffness, thus with or without horizontal forces. This allows us to test in the fixed distal end (FDE) and unfixed distal end (UDE) configurations as found in relevant literature [7-9], together with other intermediate configurations (Figure 4).

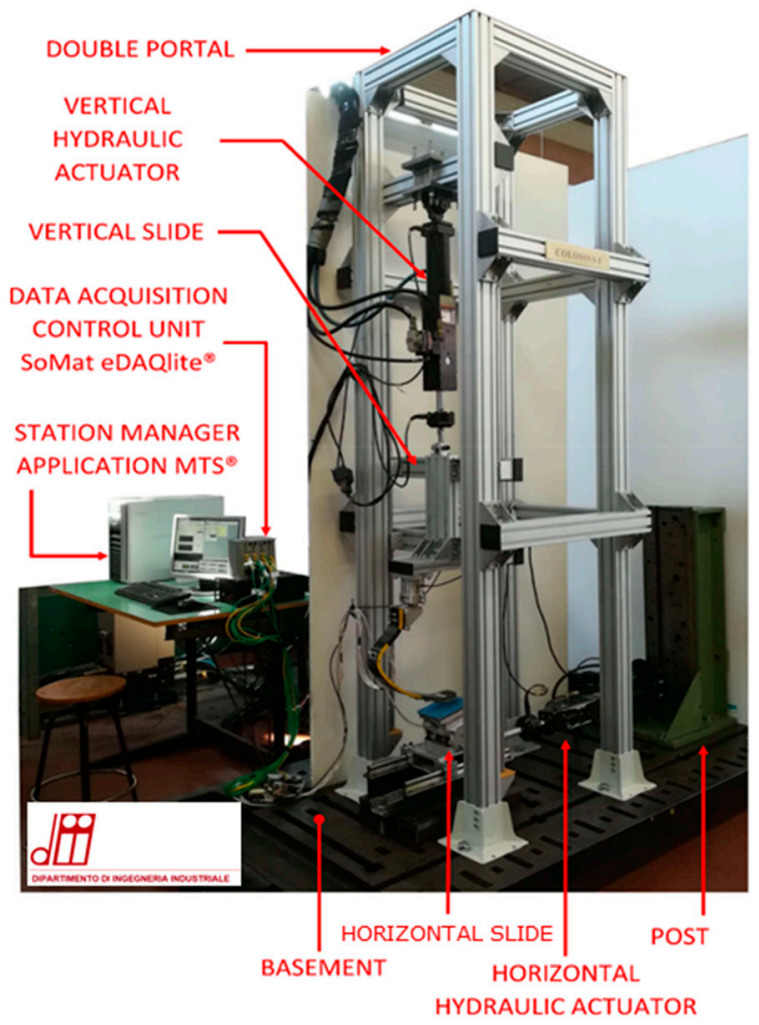

Figure 3. The multicomponent test bench developed at the Department of Industrial Engineering, University of Padova, with indication of actuators parallel (vertical) and normal (horizontal) to the socket/pylon axis. 


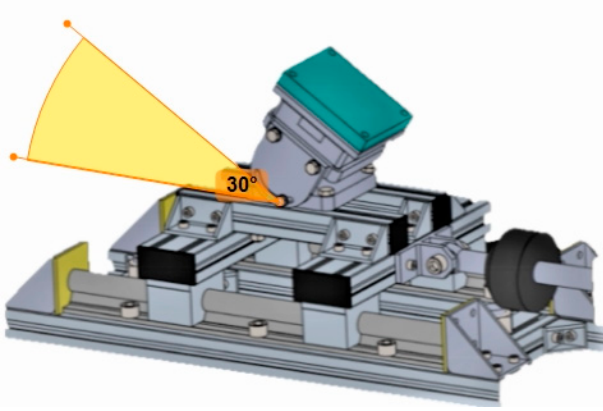

(a)

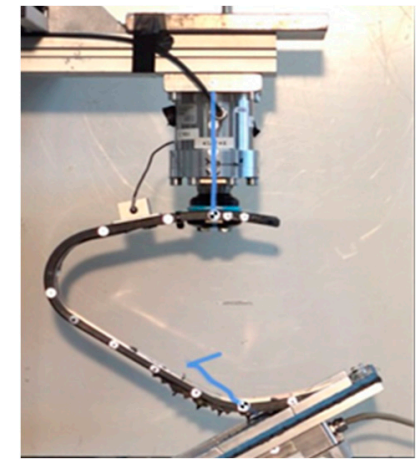

(b)

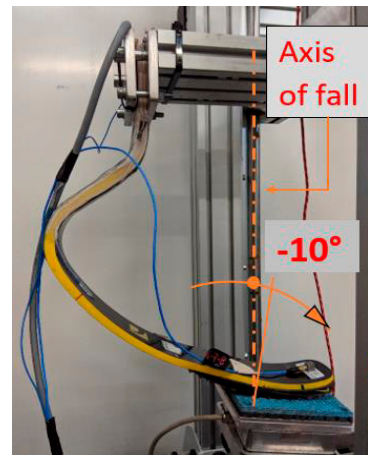

(c)

Figure 4. Detailed view of the horizontal slide of the bench and running prosthetic feet (RPFs) under test: (a) Horizontal slide with swiveling plane covered with tartan supported by a triaxial load cell, oriented at $-30^{\circ}$ from the socket axis; (b) running prosthetic foot (Ottobock Runner Cat 3) under test in the multi-component-prostheses-test-bench. Upper 6-axis load cell and lower 3-axis load cell are in evidence, with the surrogate ground oriented at $+30^{\circ}$ from the socket axis. (c) Setup of the drop test on Ossur Cheetah Cat5 with surrogate ground oriented at $-10^{\circ}$ from the socket axis.

The vertical hydraulic cylinder can be actuated at high speed, with load cycles from 0 to 6000 to $0 \mathrm{~N}$ with a period of $0.12 \mathrm{~s}$, corresponding to the load and timing typical of the sprinting stance phase and long jumping take off. Multiple-cycles tests can be performed on RPFs to evaluate their fatigue strength and prevent field failures, which can possibly result in severe injuries for the athlete.

Finally, the bench test can be re-configured to perform the dynamic drop test on the surrogate ground floor by removing the hydraulic vertical actuator and applying a lift and release system to the vertical slide. The vertical slide can be equipped with a set of appropriate dead weights and released from an appropriate drop height to replicate the GRFs intensity and the ground contact durations measured in vivo during running or jumping (Figure 4c).

Static, cyclic, and impact tests were performed on commercially available RPF with different alignments, load combinations, and ground inclinations. Specifically, the bench was first applied to test the Ottobock 1E91 Standard Runner cat3 foot. A static test was completed to study three alignments (clamp at $+4^{\circ}, 0^{\circ}$, and $-4^{\circ}$ ), three load combinations (factor $\mathrm{Q}=\mathrm{Fxs} / \mathrm{Fys}$ set to $-0.2,0,+0.2$ ), and four ground inclinations $\left(\theta=-15^{\circ}, 0^{\circ},+15^{\circ},+30^{\circ}\right)$. Theta is the absolute angle in the sagittal plane between the prosthetic pylon/socket axis and the ground (negative at heel strike, null around midstance, positive at take-off).

Secondly, two feet, the Ottobock 1E91 Runner Custom cat3 (C-shaped) and the Ossur Cheetah Xtreme cat5 (J-shaped), were compared during dynamic tests after the application of $3500 \mathrm{~N}$ in 0.12 s. Finally, the Ossur Cheetah Xtreme cat5 also underwent drop tests at ground angles ranging from $-15^{\circ}$ to $40^{\circ}$, with a drop height of $0.226 \mathrm{~m}$ and a total slide mass of $18.5 \mathrm{~kg}$, corresponding to a drop energy of $41 \mathrm{~J}$.

\section{Results}

Figure 5 reports the results of the static stiffness tests on the Ottobock 1E91 Standard Runner cat3. In Figure 5a the effect of Ottobock clamp location along the proximal portion of the foot is shown: For the load range of $0-1500 \mathrm{~N}$, small differences are evident between position $+4^{\circ}$ and position $-4^{\circ}$. The effect of load ratio Q is evident, as shown in Figure 5b: In fact, the presence of a loading component Fx opposing the RPF natural tendency of sliding forward while deflecting vertically (that correspond to $\varrho=-0.2$ ) increases the stiffness by about $35 \%$ with respect to the UDE condition ( $\varrho=-0.2)$. The largest effect in the stiffness curve is obtained when the foot-ground inclination angle $\theta$ changes from $-15^{\circ}$ ("heel strike") to $30^{\circ}$ ("Toe off"). 


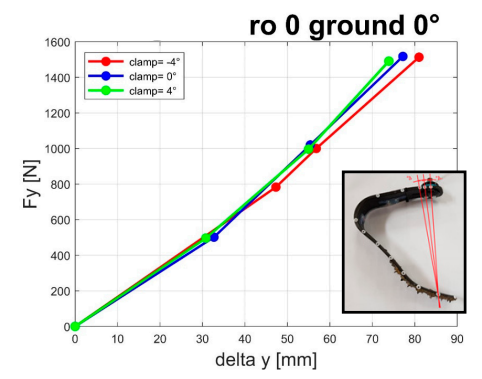

(a)

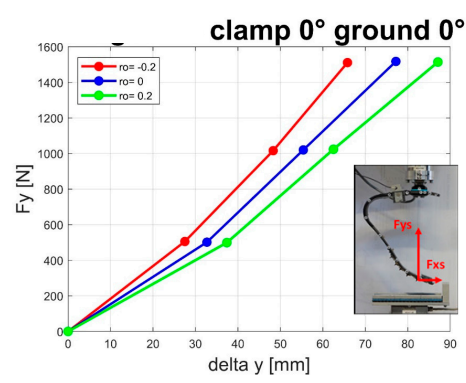

(b)

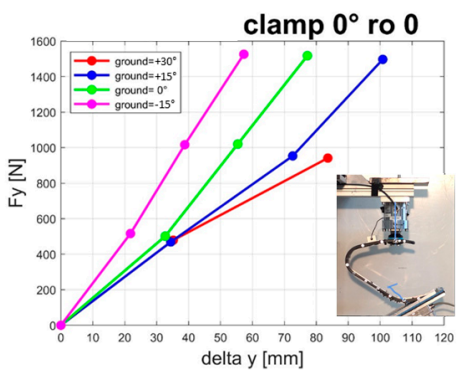

(c)

Figure 5. Results of static stiffness tests on Ottobock 1E91 Standard Runner Cat 3: (a) The effect of clamp location along the proximal portion of the foot; $(\mathbf{b})$ the effect of load ratio ( $\mathrm{Q}=$ ro) between normal Fxs and parallel Fys forces; (c) the effect of foot-ground inclination angle $\theta$ on the stiffness curve.

Results of dynamic tests performed on the C-shaped Ottobock 1E91 Custom Runner cat3 and Jshaped Ossur Cheetah Xtreme cat5 during dynamic stiffness tests are reported in Figure 6a. The two RPF load-deflection curves exhibit a bilinear behavior, with a slope change at around $1500 \mathrm{~N}$ for both. The stiffening of the two feet is quite different, with the Ottobock foot reaching a deflection $160 \%$ larger than the Ossur deflection at the peak load of $3500 \mathrm{~N}$.

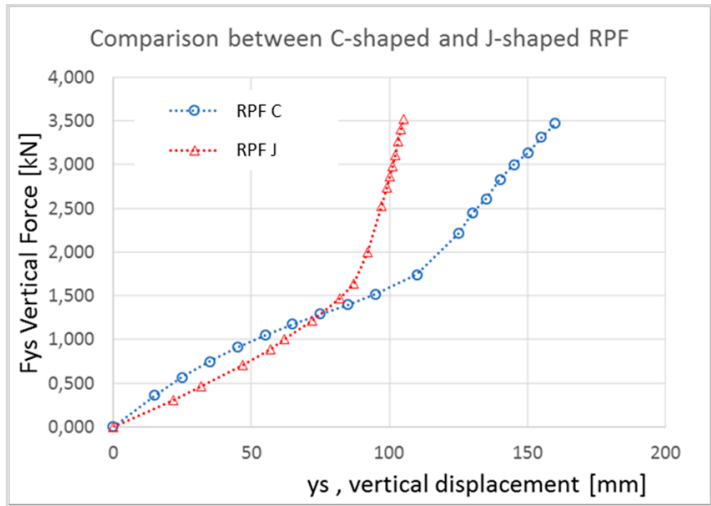

(a)

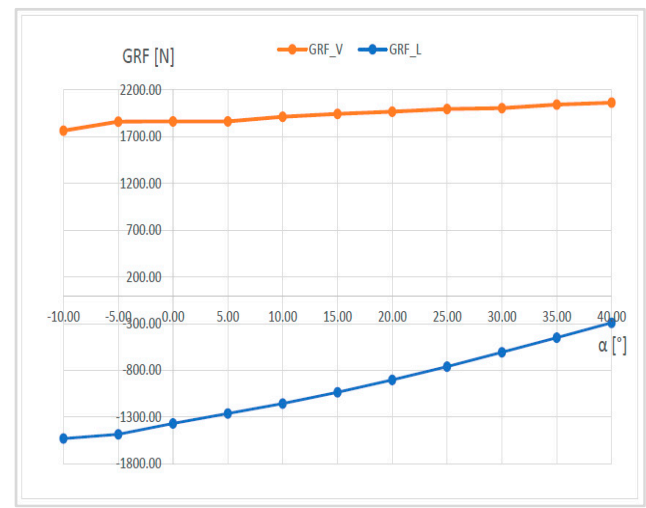

(b)

Figure 6. Results of cyclic and impact tests on different RPF: (a) Comparison of C-shaped Ottobock 1E91 Custom Runner cat3 with J-shaped Ossur Cheetah Xtreme cat5 during dynamic stiffness tests; (b) the effect of ground inclination on the force components resolved in the global reference frame developed after a $40 \mathrm{~J}$ impact drop test on Ossur Cheetah Xtreme cat5.

Results of drop tests performed on the J-shaped Ossur Cheetah Xtreme cat5 are shown in Figure $6 \mathrm{~b}$. The force components Fxg and Fyg are the forces expressed in the global reference frame that are reported in Figure 2a: If the foot is dropped against the ground inclined at increasing angles, it shows a tendency to progressively reduce the horizontal global component FxG while slowly increasing the vertical component FyG. This means that the foot seems to have a possible "neutral" drop direction, likely around $\mathrm{a}+45^{\circ}$ angle, where the ground reaction generated by the drop is parallel to the drop direction. 


\section{Discussion}

The aim of the work was to conceive, design, construct, and preliminary test a multicomponent test bench for the structural testing of the running prosthetic feet under static, dynamic, and impact test conditions.

The bench concept adopted the RPF clamp axis as a vertical axis, as most of the existing test setups that can be found in literature [7-9]. However, the first original contribution was the design of a specific test frame with a double portal rather than adopting universal test machines with accessories for the simulation of foot/ground interaction. The second difference with the existing test benches was the introduction of a second cylinder for the application of controlled loads normal to the socket/pylon axis. This combination of loads has not yet been applied in the literature. Similarly to former studies, the bench allows us to reproduce the known ranges of normal Fxs and parallel Fys force combinations recorded in vivo (expressed by load ration @) ranging from the UDE restraint condition $(\varrho=0)$ to the FDE $(\varrho<0)$ [7]. In particular, the latter condition occurs when the RPF is highly loaded against the ground, but its tip is prevented from any motion normal to the socket ys axis. The analysis of loads resolved in the socket reference frame, as shown in Figure $2 d-f$, shows that, for instance, at $20 \%$ stance, the ratio between normal and parallel load components is about +0.2 . Further experiences performed by the authors in field tests [11] confirmed that $\varrho$ reaches a maximum value of +0.2 during the early stance and tends to decrease to 0 at the midstance and afterward. This states that the UDE conditions, typically resulting in highly negative $\varrho$ values, are unlikely to be reached by the RPF during real running events.

Finally, the use of drop tests at fixed ground angles, despite apparently similar to the real use during in vivo field tests, is possibly a more conventional comparative method that still needs a deeper understanding to match with the fast dynamic orientation changes that the foot experiences in reality within $0.12-0.15 \mathrm{~s}$ of stance $[10,11]$.

\section{Conclusions}

The present work presents the concept, design, construction, and implementation of a multicomponent test bench for the characterization of running prosthetic feet under static, dynamic, and impact conditions. The bench enabled comparative tests on RPF to be performed at different alignments, ground inclinations, and load combinations. Tests performed on C-shaped and J-shaped RPFs allowed us to compare different feet and to explore the effect of different factors on the loaddeflection curves under static, dynamic, and impact conditions. The bench allows further in-depth investigations to establish which test methods will better represent the real running kinetics on the foot.

Acknowledgments: Authors thank INAIL for supporting the research.

Conflicts of Interest: The authors declare no conflict of interest.

\section{References}

1. Nolan, L. Carbon fibre prostheses and running in amputees: a review. Foot Ankle Surg. 2008, 14, 125-129.

2. Brüggemann, G.-P.; Arampatzis, A.; Emrich, F.; Potthast, W. Biomechanics of double transtibial amputee sprinting using dedicated sprinting prostheses. Sports Technol. 2008, 1, 220-227.

3. Grabowski, A.M.; McGowan, C.P.; McDermott, W.J.; Beale, M.T.; Kram, R.; Herr, H.M. Running-specific prostheses limit ground-force during sprinting. Biol. Lett. 2009, rsbl20090729.

4. Weyand, P.G.; Bundle, M.W.; McGowan, C.P.; Grabowski, A.M.; Brown, M.B.; Kram, R.; Herr, H. The fastest runner on artificial legs: different limbs, similar function? J. Appl. Physiol. 2009, 107, 903-911.

5. Mcgowan, C.P.; Grabowski, A.M.; McDermott, W.J.; Herr, H.M.; Kram, R. Leg stiffness of sprinters using running-specific prostheses. J. R. Soc. Interface 2012, rsif20110877.

6. Hobara, H.; Baum, B.S.; Kwon, H.-J.; Miller, R.H.; Ogata, T.; Kim, Y.H.; Shim, J.K. Amputee locomotion: Spring-like leg behavior and stiffness regulation using running-specific prostheses. J. Biomech. 2013, 46, 2483-2489.

7. Dyer, B.T.J.; Sewell, P.; Noroozi, S. How should we assess the mechanical properties of lower-limb 
prosthesis technology used in elite sport? An initial investigation. J. Biomed. Sci. Eng. 2013, 6, 116-123.

8. Grobler, L. Characterisation of Running Specific Prostheses and its Effect on Sprinting Performance. Ph.D. Thesis, Stellenbosch University, Stellenbosch, South Africa, 2015.

9. Beck, O.N.; Taboga, P.; Grabowski, A.M. Characterizing the mechanical properties of running-specific prostheses. PLOS ONE 2016, 11, e0168298.

10. Makimoto, A.; Sano, Y.; Hashizume, S.; Murai, A.; Kobayashi, Y.; Takemura, H.; Hobara, H. Ground reaction forces during sprinting in unilateral transfemoral amputees. J. Appl. Biomech. 2017, 33, 406-409.

11. Petrone, N.; Costa, G.; Foscan, G.; Gri, A.; Boekestijin, R.; Migliore, G.; Cutti, A.G. Collection of Structural Loads Acting on Instrumented Running Specific Prostheses during Field Tests on Elite Atletes. In Proceedings 2020 MDPI; MDPI: Basel, Switzerland, 2020

(C) 2020 by the authors. Licensee MDPI, Basel, Switzerland. This article is an open access article distributed under the terms and conditions of the Creative Commons Attribution (CC BY) license (http://creativecommons.org/licenses/by/4.0/). 